\title{
Malignant Neoplasm of the Thoracic Esophagus
}

National Cancer Institute

\section{Source}

National Cancer Institute. Malignant Neoplasm of the Thoracic Esophagus. NCI

Thesaurus. Code C3532.

A primary or metastatic malignant neoplasm involving the thoracic region of the esophagus. 